\title{
Some results of experimental investigation of super-deep penetration into the metal's targets
}

\author{
S.K. Andilevko, O.V. Roman, S.M. Usherenko and V.A. Shilkin
}

Belorussian Research and Production, Powder Metallurgy Association, 220600 Minsk, Platonov str. 4I, Belarus

\begin{abstract}
The phenomenon of Super-Deep Penetration (SDP) is briefly presented. Various experimental techniques, including that based on neutron-activated autoradiography (NAAR), for experimentally investigating this phenomenon are described. Results from a number of investigations in which the effect on SDP of initial target temperature, the dependence of the concentration on depth for different powder particles and the use of bimetallic targets, are reviewed. The complicated nature of the SDP process and the need for a wider range of experimental data if it is to be fully understood are discussed.
\end{abstract}

\section{INTRODUCTION}

The Super-Deep Penetration (SDP) effect which is being studied here involves some basic principles which characterise modern physical and chemical theories concerned with the behaviour of colliding solids. SDP is the penetration of discrete particles to depths $\geq 10^{3} d_{p}$, where $d_{p}$ is the initial diameter of the particles and $d_{p} \leq 100 \mu \mathrm{m}$, under conditions where a dense powder flux, of density $\rho_{F} \approx 2 \mathrm{~g} / \mathrm{cm}^{3}$, interacts at high velocity, $U_{F} \approx 2 \mathrm{~km} / \mathrm{s}$, with a metallic target. Various methods in which different metallic targets were studied have been used by researchers to obtain information about SDP. We will describe here some of these investigations.

\section{EXPERIMENTAL METHODS}

\subsection{Neutron-Activated Autoradiography}

An initial method used in many investigations is that of neutron-activated autoradiography (NAAR) to study targets treated by a powder flux. NAAR is a well-established method to investigate local chemical and structural inhomogeneities in materials. It allows the detection of sources of ionization (those indicating the presence of chemical elements and their compounds) using the 
image of their field of doses (autoradiogramm, AR). In these experiments $B$ and its compounds were injected into iron targets. To investigate the behaviour of $B$ in the target under SDP the NAAR method of track's (TNAAR) was used to detect the nuclear reaction $10 B(n, a)^{7} \boldsymbol{L} i$, which has an anomalously high section of reaction under the action of high temperature neutrons. To detect $\alpha$-particles $\left({ }^{7} \mathrm{Li}\right.$ is not detected) a thin film, $10 \mu \mathrm{m}$ thick, of $\boldsymbol{C}_{12} \mathrm{H}_{18} \mathrm{O}_{7}$ was used. After irradiation of a penetrated sample by high temperature neutrons the film was separated from the sample and chemically treated, using a special method. The content of elements in the samples was measured through the density of tracks on the AR. The concentration of $B$ was determined from the equation $C_{B}=K \rho / \Psi$, where $\rho$ is the average density tracks, $\Psi$ is a function of the influence of high temperature neutrons, $\boldsymbol{K}$ is a special geometrical constant relating to a known autoradiographical situation (for this case $\boldsymbol{K} \sim 0.75 \times 10^{-4}$ ). This method was used for investigation of $\mathrm{Fe}, \boldsymbol{T i}$ and $\mathrm{Cu}$ samples which had been cut from the cylindrical samples along the direction of motion of the $B$ particles, see fig. 1 .

Autoradiographs (ARs) of iron samples, see fig. 2, showed the presence of $B$ in a fibre-like formation with a strongly varying concentration (for $C_{B}$, in mass per cent., from $\approx 5.6 \times 10^{-4}$ to $\geq 10^{-1}$ ) in both the transverse and the longitudinal directions. In the initial iron samples, before SDP, the average $B$ concentration did not exceed $C_{B} \approx 10^{-4}$ mass per cent. and all $B$ formations were situated on the grain boundaries. After SDP the situation has essentially changed. The $\boldsymbol{B}$ formations were observed at any point throughout the samples. The highest density of $\boldsymbol{B}$ formations were detected near $t$ he axis of the powder flux impacting the target. The fibre-like formation of $\boldsymbol{B}$ inclusions illustrates the particle motion within the target. They are situated along the direction of propagation of the powder flux and their concentration increases towards the axis of the flux. In our opinion, which is also based on the investigation of these samples by other methods such as chemical analysis, electron microscopy etc., these $\boldsymbol{B}$ inclusions are very small parts of particles which are left behind by the particle during its motion through the target (the process of mass losing).

\subsection{Effect of Initial Target Temperature}

The second cycle of experiments was devoted to the investigation of the influence of the initial temperature of the iron target on the SDP process. The target was given a preliminary anneal at a temperature of $950^{\circ} \mathrm{K}$ for 1 hour. The target was then subjected to a flux of $\mathbf{T i B}_{2}$ powder for which $40 \mu \mathrm{m} \leq d_{p} \leq 6 \mu \mathrm{m}$. The chemical analysis (CA) of a $B$ inclusion was performed using an atomic emission spectrometer (AES). After analysis of the results obtained over the whole of the temperature range investigated, three specific thermal zones were identified:

- A low temperature zone, from $77^{\circ}$ to $200^{\circ} \mathrm{K}$, showing a local maximum at a temperature below $150^{\circ} \mathrm{K}$.

- A middle temperature zone, from $200^{\circ}$ to $900^{\circ} \mathrm{K}$, in which the fluctuations in the concentration of $\boldsymbol{B}$ are very small, less than the experimental scatter.

- A high temperature zone, from $900^{\circ}$ to $1160^{\circ} \mathrm{K}$, within which a special local maximum is observed at a temperature of $1040^{\circ} \mathrm{K}$.

In order to estimate the dependence of these results on the chemical composition of the powder used some special experiments were performed in which the target was treated with a powder flux of $C o$ particles for which $d_{p} \leq 40 \mu \mathrm{m}$. Again three temperature ranges were observed: $77^{\circ}$ to $220^{\circ} \mathrm{K}$, $220^{\circ}$ to $250^{\circ} \mathrm{K}$ and $250^{\circ}$ to $1250^{\circ} \mathrm{K}$. Such local maxima may be explained if at temperatures of $130^{\circ} \mathrm{K}, 1040^{\circ} \mathrm{K}$ and $1180^{\circ} \mathrm{K}$ the target material is in a state of structural instability. 
C8-805

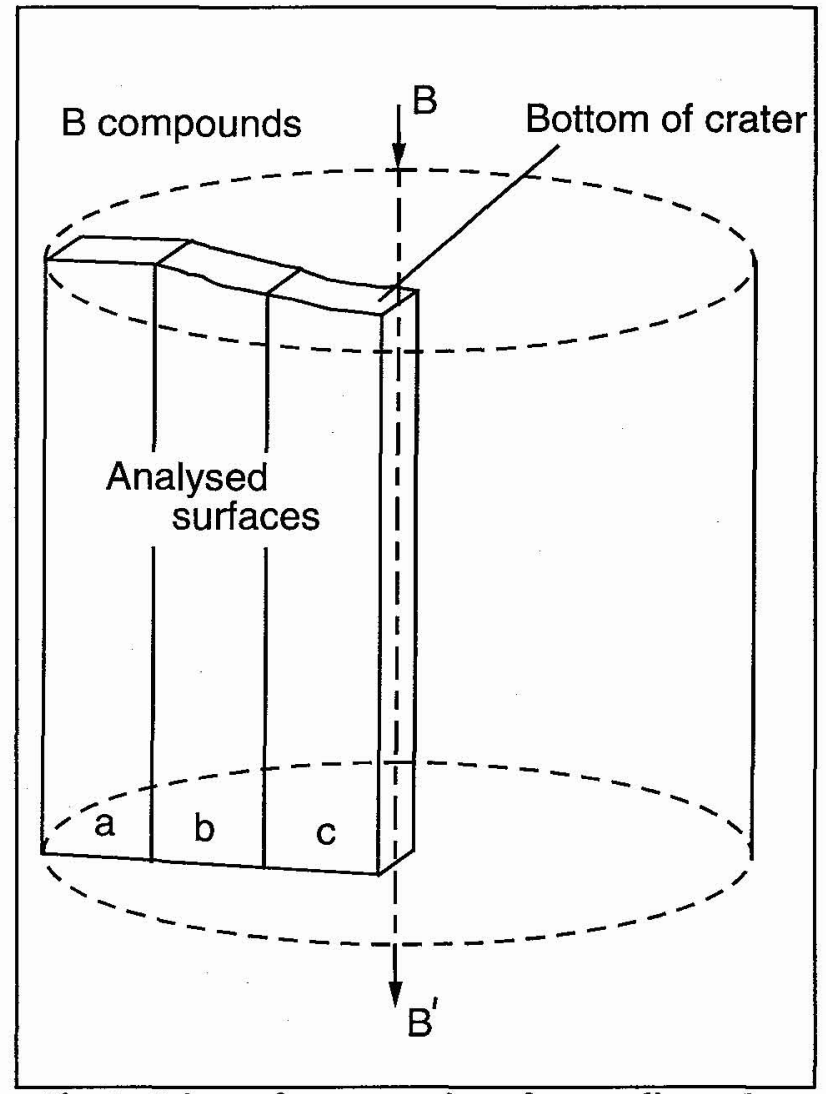

Fig. 1 Scheme for preparation of autoradiography samples

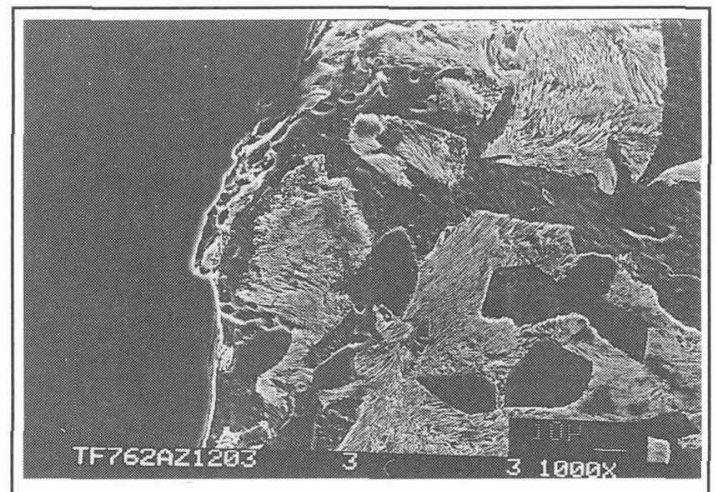

Fig. 3 Structure of the Fe - Ti interface after loading with a B compound powder flux 


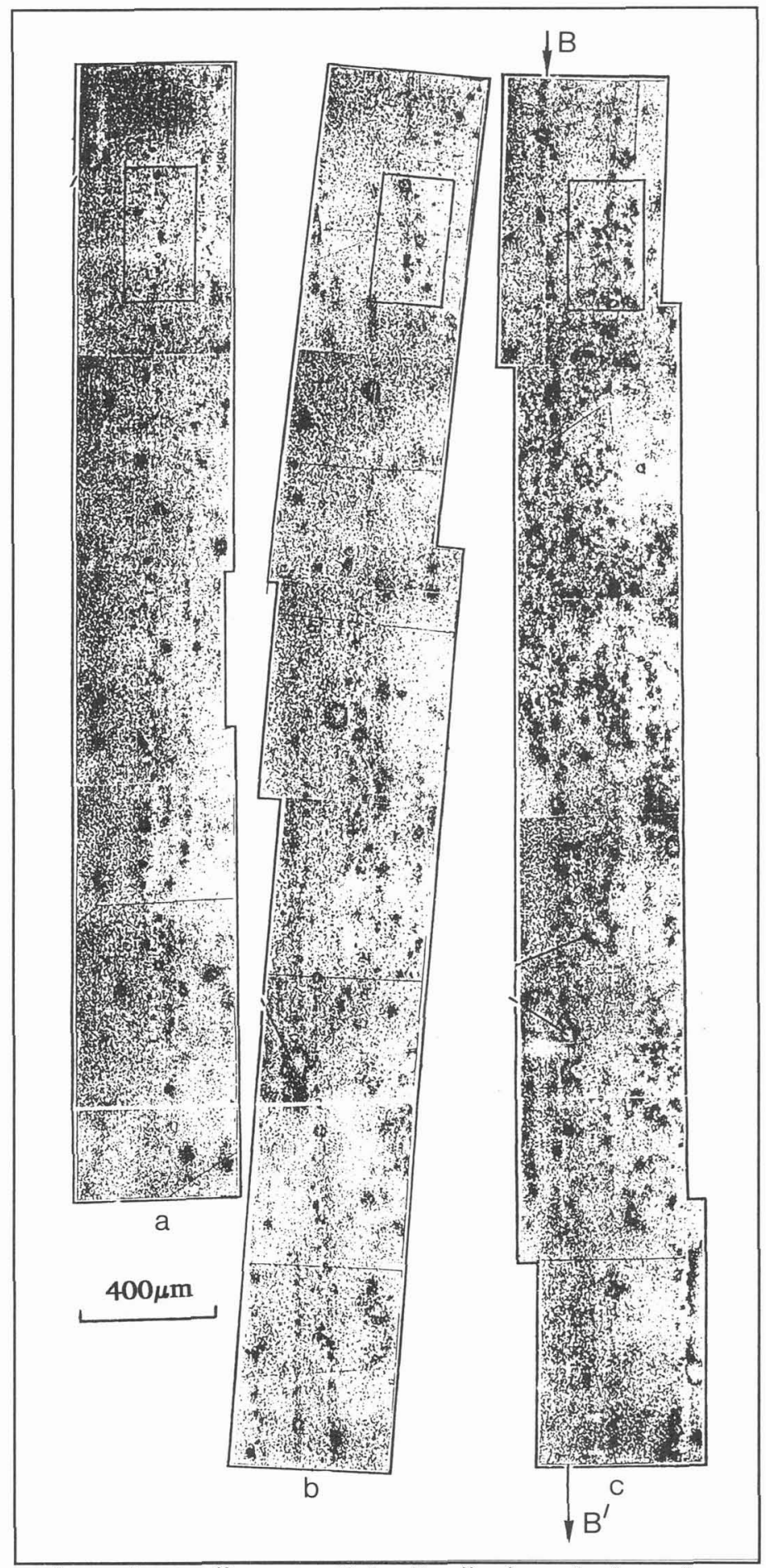

Fig. 2 Autoradiograms of the distribution of $B$ in an iron sample (analysed surfaces - a, b and c - correspond to those in fig. 1) 


\subsection{The Effect of Different Powder Particles}

In a third cycle of experiments a more comprehensive study was made of the effect of different powders particles on the variation of concentration with the depth of penetration. For these investigations powders of $\mathrm{Ti}, \mathrm{Cr}, \mathrm{Mo}, \mathrm{NB}, \mathrm{C}, \mathrm{B}, \mathrm{S}, \mathrm{TiB}, \mathrm{NbB}$ etc. were used. Iron samples were used as targets in these experiments. The inclusions were studied using a layer by layer chemical analysis method. As a rule the variation of concentration with depth could be followed approximately by an equation of the form:

$$
N=A h^{3}+B h^{2}+C h+D
$$

where $\boldsymbol{N}$ is the concentration, $\boldsymbol{h}$ is the depth and $\boldsymbol{A}, \boldsymbol{B}, \boldsymbol{C}$ and $\boldsymbol{D}$ are the coefficients of the equation. All powders used in these experiments showed the same behaviour.

\subsection{Bimetallic Targets}

And, finally, the fourth cycle of experiments was devoted to the investigation of SDP in bimetallic targets. For this purpose the target was a $T i$ sample attached to the bottom of an iron sample. To allow for the effect of the interface a comparison was made with the results for targets where an iron sample was attached to the bottom of a second iron sample. The attaching of samples was performed by the method of explosive welding and the examination of the inclusions was achieved by the methods of micro $X$-ray spectral analysis and neutron radiography. In previous experiments it had been established that SDP of $\boldsymbol{B}$ compounds into $\boldsymbol{T} \boldsymbol{i}$ targets is not observed. Observation of the collision of $\boldsymbol{B}$ compounds with $\boldsymbol{T i}$ targets in air is impossible due to the complications of the powder particles flux. It was proposed, therefore, to make the top sample of iron in the bimetallic $\mathrm{Fe}$ - $\mathrm{Fi}$ target, allowing the motion of the particles to be established before interaction with the surface of the $\boldsymbol{T i}$ sample (analogous to Wilson's camera in nuclear physics). When the $\boldsymbol{F e}-\boldsymbol{F e}$ target was loaded with a $\boldsymbol{B}$ compound powder flux the $\boldsymbol{B}$ inclusions were detected in both $\boldsymbol{F e}$ samples. However, when $\boldsymbol{F e}-\boldsymbol{T} \boldsymbol{i}$ targets were loaded by the $\boldsymbol{B}$ compound powder flux the $\boldsymbol{B}$ inclusions were only detected in the $\boldsymbol{F e}$ sample and were not observed in the $\boldsymbol{T i}$ sample.

More detailed investigation of the local region near the $\boldsymbol{F e}-\boldsymbol{T i}$ interface revealed an interesting behaviour. Particles, with $d_{p} \approx 10 \mu \mathrm{m}$, which were moving in the $F e$ sample during SDP began to change their direction of motion as they approached within a distance of around $40 \mu \mathrm{m}$ before the interface with the $\boldsymbol{T i}$ sample (Fig. 3) and started to follow an approximately circular curve with a radius of around $60 \mu \mathrm{m}$. A direct contact of the particle with the surface of the $\mathrm{Ti}$ sample was not observed during all the time of motion, all particles stopping if they reach the $\mathrm{Fe}-\mathrm{Ti}$ interface. The specific energy spent on changing the particle direction of motion was about $5 \times 10^{5} \mathrm{~J} / \mathrm{kg}$. The mechanism of such particle behaviour near the interface is unknown. However, the fact that this effect is absent when a $\mathbf{F e}$ - $\mathbf{T i}$ bimetallic target is loaded by a $\mathbf{S i}$ compound powder flux allows us to assume that in this case the mechanism of chemical selection of SDP take place.

\section{CONCLUSIONS}

The results of these various methods of investigating SDP all show the complicated character of the behaviour of the penetrating particles as they move within the target material. The concentration of elements penetrating into the target depends on a lot of factors, e.g. chemical composition of powders and targets, atomic activity of particles, temperature of the target and presence of interfaces. The distribution of particles in the target is non-linear in character. In our opinion the penetration of $\boldsymbol{B}$ compound powders may be determined by the process of particle destruction (erosion) during penetration of the powder flux.

The experimental data described above represent only a small part of the investigations which are likely to be necessary for a full understanding of the mechanics of SDP. 